\title{
Assessment of bone densitometry using radiography with a step-wedge phantom: a pilot study of the forearm
}

\author{
Ling Wang ${ }^{1,2 \#}$, Pengju Huang ${ }^{1 \#}$, Hui Du ${ }^{3 \#}$, Jian Geng ${ }^{1}$, Xinghua Yin ${ }^{3}$, Yandong Liu ${ }^{1}$, Tanuj Puri ${ }^{4}$, Bo $\mathrm{He}^{5}$, \\ Liang Lyu ${ }^{2}$, Xiaoguang Cheng ${ }^{1}$, Xieyuan Jiang ${ }^{6}$, Klaus Engelke $^{7,8}$, Glen M. Blake ${ }^{4}$ \\ ${ }^{1}$ Department of Radiology, Beijing Jishuitan Hospital, Beijing, China; ${ }^{2}$ Department of Radiology, The First People's Hospital of Yunnan Province, \\ Kunming, China; ${ }^{3}$ Department of Orthopedics, Beijing Jishuitan Hospital, Beijing, China; ${ }^{4}$ School of Biomedical Engineering \& Imaging Sciences, \\ King's College London, St Thomas' Hospital, London, UK; ${ }^{5}$ Department of Radiology, The First Affiliated Hospital of Kunming Medical \\ University, Kunming, China; ${ }^{6}$ Department of Traumatic Orthopedics, Beijing Jishuitan Hospital, Beijing, China; ${ }^{7}$ Department of Medicine 3 , FAU \\ University Erlangen-Nürnberg and Universitätsklinikum Erlangen, Erlangen, Germany; ${ }^{8}$ Institute of Medical Physics, University of Erlangen, \\ Erlangen, Germany
}

Contributions: (I) Conception and design: L Wang, K Engelke, X Cheng, X Jiang; (II) Administrative support: P Huang, H Du, J Geng; (III) Provision of study materials or patients: L Wang, B He, L Lyu, P Huang, Y Liu, H Du, X Yin; (IV) Collection and assembly of data: P Huang, H Du, J Geng; (V) Data analysis and interpretation: L Wang, GM Blake, K Engelke; (VI) Manuscript writing: All authors; (VII) Final approval of manuscript: All authors.

"These authors contributed equally to this work.

Correspondence to: Xiaoguang Cheng. Department of Radiology, Beijing Jishuitan Hospital, 31 Xinjiekou East Street, Beijing 100035, China. Email: xiao65@263.net; Xieyuan Jiang. Department of Traumatic Orthopedics, Beijing Jishuitan Hospital, 31 Xinjiekou East Street, Beijing 100035, China.Email: jxytrauma@163.com.

Background: Radiographic absorptiometry (RA) is one of the earliest methods of bone densitometry and has been used to measure the phalanges and metacarpals where soft tissue attenuation is minimal. The aim of this study was to determine whether the technique can be adapted to correct for soft tissue attenuation and measure areal bone mineral density (aBMD) in the forearm.

Methods: A total of 51 patients referred for a clinical spine and hip dual-energy X-ray absorptiometry (DXA) examination and 8 young and middle-aged volunteers were recruited to this study. The first 29 participants (20 women, 9 men, aged $61 \pm 14$ years) served as the training cohort, and the remaining 30 (20 women, 10 men, aged $55 \pm 16$ years) comprised the validation cohort. All participants underwent a DXA scan of their non-dominant forearm, and a digital X-ray image of the same arm was acquired with a step phantom. Identical regions of interest (ROIs) in the radius and ulna at the one-third radius site were measured on the X-ray and DXA images, and a soft tissue ROI was measured on X-ray images between the radius and ulna. The X-ray measurements in the training cohort were expressed as equivalent step phantom thickness (Eq. SPT) and used to estimate forearm aBMD using a linear equation calibrated against the DXA scans. Estimates of forearm aBMD made from the digital X-ray images acquired in the validation cohort were compared with the results of the DXA scans.

Results: Digital X-ray estimates of radius and ulna aBMD at the one-third radius site in the validation cohort showed a good correlation with GE-Lunar iDXA scanner measurements $(r=0.795 ; \mathrm{P}<0.001)$. The Bland-Altman plot had a mean bias of $-0.002 \mathrm{~g} / \mathrm{cm}^{2}$ and $95 \%$ limits of agreement of -0.185 to $+0.181 \mathrm{~g} / \mathrm{cm}^{2}$. Conclusions: Digital X-ray estimates of proximal forearm aBMD corrected for soft tissue attenuation correlated with DXA measurements with correlation coefficients comparable to those seen for other peripheral bone densitometry technologies. 
Keywords: Radiographic absorptiometry (RA); dual-energy X-ray absorptiometry (DXA); bone mineral density (BMD); forearm

Submitted Aug 25, 2021. Accepted for publication Jan 11, 2022.

doi: $10.21037 /$ qims-21-842

View this article at: https://dx.doi.org/10.21037/qims-21-842

\section{Introduction}

In increasingly ageing societies, the incidence of osteoporotic fracture is rising. However, osteoporosis is often under-diagnosed in elderly populations worldwide. Bone mineral density (BMD) is widely used in predicting fracture risk and routinely applied for recognizing osteoporosis. Dual-energy X-ray absorptiometry (DXA) is widely used in clinical settings to measure areal BMD (aBMD) in the spine, hip, and forearm. However, access to the DXA examinations varies geographically and is often insufficient. In China, there are only 0.46 DXA systems per million inhabitants (1), which does not adequately meet the diagnostic needs of most of the Chinese population (2).

Although the International Society for Clinical Densitometry (ISCD) recommends the use of forearm DXA scans only when the hip and/or spine cannot be measured or interpreted for technical reasons, forearm aBMD assessments are effective at predicting major osteoporotic fractures $(3,4)$, for studies of bone growth $(5)$, the effects of exercise (6), and in clinical trials $(7,8)$. Furthermore, forearm aBMD is particularly effective at predicting the risk of wrist and forearm fractures (9).

Radiography is the most widely available, noninvasive technique for visualizing bone (10). However, radiographs are relatively insensitive to changes in aBMD. For example, it has been estimated that up to $20-40 \%$ of bone mass must be lost before a decrease in the visual density of bone can be seen on lateral spine radiographs (11). Quantitative techniques such as radiographic absorptiometry (RA) attempt to estimate aBMD from the optical density of X-ray images beyond the visual interpretation of radiographs. However, due to imprecision from factors related to image acquisition such as beam hardening, overlying soft tissue, and calibration in arbitrary units such as equivalent aluminum thickness, RA techniques have limited accuracy for aBMD assessment and are mainly confined to measurements of the phalanges and metacarpals where soft tissue attenuation is minimal.

In this study we evaluated the use of a step phantom combined with a digital $\mathrm{X}$-ray-device and proposed a new method to quantify aBMD of the forearm based on radiographic imaging with a correction for soft tissue attenuation. The study was planned as a pilot study for a project to use the same $\mathrm{X}$-ray device to evaluate bone age in 20,000 children across China and to include the step phantom to simultaneously evaluate forearm aBMD. We present the following article in accordance with the Transparent Reporting of a multivariable prediction model for Individual Prognosis or Diagnosis (TRIPOD) reporting checklist (available at https://qims.amegroups.com/article/ view/10.21037/qims-21-842/rc).

\section{Methods}

\section{Participants}

The study was conducted in accordance with the Declaration of Helsinki (as revised in 2013), approved by the institutional review board of the principal investigator's hospital, and all participants provided their written informed consent. A training cohort of 29 participants (20 women and 9 men, aged 45-79 years) attending the hospital for a routine clinical spine and hip DXA examination were enrolled between May and June 2020 (Table 1). Another 30 participants ( 20 women and 10 men, aged $25-83$ years) were recruited in August 2020 for the validation cohort. With the exception of 8 young to middle-aged participants, the participants in the validation cohort were also patients referred for a clinical DXA examination. Potential participants were referred to the study by their DXA technician. A radiologist explained the study to the patient and asked them if she/he was willing to participate in the study. If they agreed to participate, she/he was asked to sign the consent form. Then, a forearm DXA scan and a forearm digital radiography image were acquired. The 8 young and middle-aged participants were investigators and their colleagues who were recruited to the validation cohort to establish a more generalized age range. Patients were excluded if they had a history of forearm fracture or wrist rheumatoid arthritis. We first used conventional DXA aBMD measurements at the one-third radius site in 
Table 1 Participant characteristics

\begin{tabular}{lccc}
\hline Characteristics & Training cohort & Validation cohort & P value \\
\hline $\mathrm{N}$ [male] & $29[9]$ & $30[10]$ & - \\
Height $(\mathrm{cm})$ & $162.3 \pm 7.2$ & $162.7 \pm 8.6$ & 0.86 \\
Weight $(\mathrm{kg})$ & $62.6 \pm 11.5$ & $62.4 \pm 10.0$ & 0.94 \\
Age (years) & $63.7 \pm 10.5$ & $55 \pm 16$ & 0.018 \\
\hline
\end{tabular}
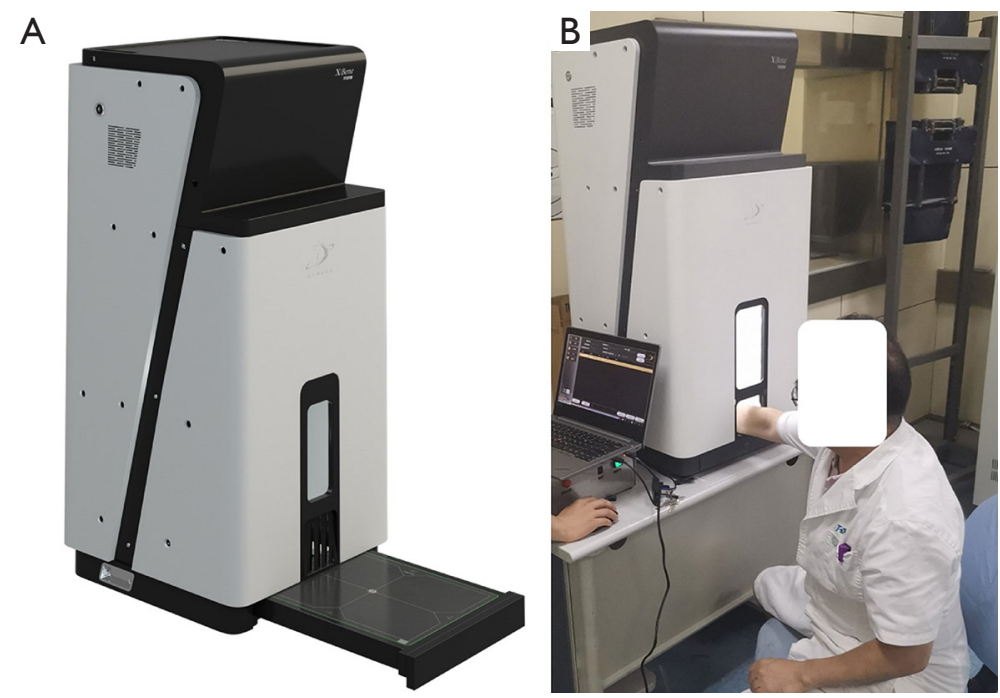

Figure 1 The Xbone digital X-ray device used for acquiring forearm images. (A) Image of the Xbone device; (B) participants sit beside the Xbone device and stretch out their forearm in the device. This image is published with the participant's consent.

the training cohort to calibrate the digital X-ray images acquired with a step phantom described in detail below. In a separate validation cohort, we compared the forearm aBMD derived from digital $\mathrm{X}$-ray images with aBMD measured by forearm DXA.

\section{Forearm imaging protocol}

Radiography and DXA were used to evaluate the nondominant forearm of all participants. Radiography images were acquired by a table mounted digital $\mathrm{X}$-ray device (Xbone, Dymena Healthcare, Shanghai, China) (Figure 1A). The participants were seated beside the Xbone machine with their forearm stretched out in the device (Figure 1B). The forearm was positioned in the image area for X-ray acquisition, and the wrist was positioned in the center of the image area identified by a cross on the imaging plate. Both the radius and ulna were straight and fully within the image area. The participant was instructed not to move. A step phantom was positioned to the left of the forearm at a distance of about $20 \mathrm{~mm}$ (Figure $2 A$ ). The step phantom (Figure $2 B$ ) was made from hydroxyapatite and soft tissue equivalent epoxy resin and was designed and manufactured specifically for this project (Radiology Quantitative Innovation Group of National Institute of Metrology, Beijing, China). The imaging parameters of the $\mathrm{X}$-ray device were: $75 \mathrm{kVp}, 200 \mu \mathrm{A}$; imaging area $300 \mathrm{~mm} \times$ $300 \mathrm{~mm}$ (image resolution: $0.12 \mathrm{~mm}$ pixels); and focus-film distance $100 \mathrm{~cm}$. The skin entrance dose was $14 \mu \mathrm{Gy}$ (12), and the effective dose for a forearm image was estimated at $0.01 \mu \mathrm{Sv}$. Forearm DXA scans were performed on a GELunar iDXA scanner (GE Lunar, Madison, WI, USA), with the participant assuming the supine position (Figure $2 C$ ) at the same time as their routine lumbar spine and proximal femur scans. A previous study showed that there is no significant difference in forearm aBMD results when the is patient positioned either in a supine or a seated position (13). The iDXA scanner X-ray tube operated at $100 \mathrm{kV}$ and 

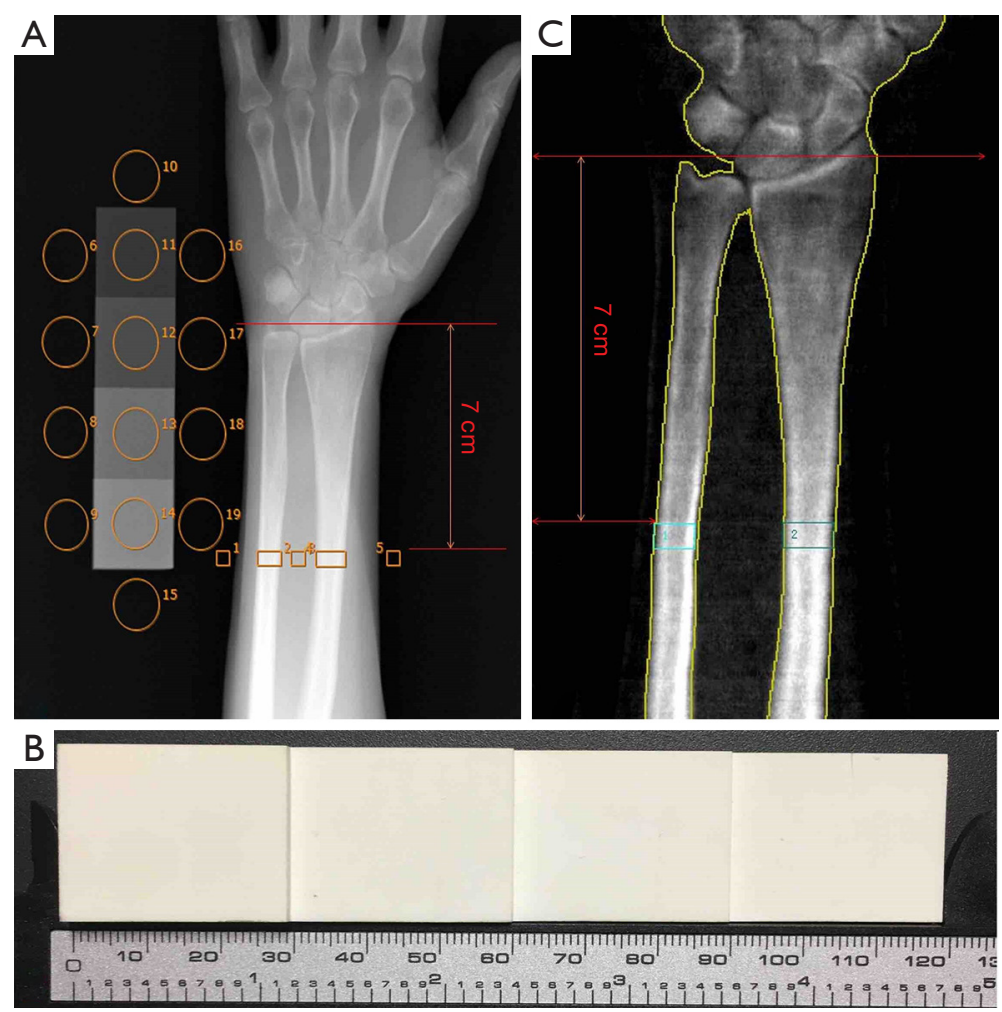

Figure 2 Xbone and DXA images of the distal forearm and an image of the 4-step phantom. (A) X-ray image of forearm and step phantom showing placement of ROIs in the phantom, background and in the forearm $70 \mathrm{~mm}$ proximal to the ulna styloid; (B) the 4-step phantom used to calibrate the X-ray images (the scale shows millimeters); (C) DXA forearm scan of the same participant showing placement of the ulna and radius ROIs. ROI, region of interest; DXA, dual X-ray absorptiometry.

a K-edge filter split the $\mathrm{X}$-ray output into high and low energy components at 70 and $38 \mathrm{keV}$, respectively. Skin entrance dose for a forearm scan was $<10 \mu \mathrm{Gy}$ (14), and the effective dose was estimated at $<0.01 \mu \mathrm{Sv}$.

\section{Data analysis}

Digital X-ray images of the forearm and step phantom were transferred to a laptop computer and analyzed using Sante DICOM viewer free software (Santesoft, Nicosia, Cyprus). The digitized pixel measurements were scaled with the logarithm of X-ray intensity. Circular regions of interest (ROI) were placed in each step of the phantom with background ROIs positioned on either side (Figure $2 A$ ). Rectangular ROIs $5 \mathrm{~mm}$ high and encompassing the full width of the radius and ulna were placed in the distal forearm $70 \mathrm{~mm}$ proximal to the tip of the ulna styloid. Further ROIs were positioned in soft tissue between the radius and ulna and as background sites either side of the forearm (Figure 2A). For DXA measurements, the GE enCORE software (GE Healthcare, Chicago, IL, USA) was used for the analyses. We used a self-defined ROI function to place 2 rectangular ROIs $5 \mathrm{~mm}$ high at the same location $(70 \mathrm{~mm}$ proximal to the tip of the ulna styloid) as we did on the digital $\mathrm{X}$-ray images to ensure consistency (Figure 2C). All analyses were performed by the same operator. Background corrected step phantom digital X-ray measurements were then plotted against step thickness (units: $\mathrm{mm}$ ) and a linear regression line was fitted (Figure 3A). Background measurements either side of the forearm were averaged and subtracted from the measurements over the radius, ulna, and the soft tissue ROIs, and the background corrected data converted into equivalent phantom thickness measurements using the step phantom calibration line. Since these measurements were taken from broad spectrum single $\mathrm{kV} \mathrm{X}$-ray images, there was no analytical equation for subtracting the soft tissue contribution to the radius and ulna ROI measurements. 

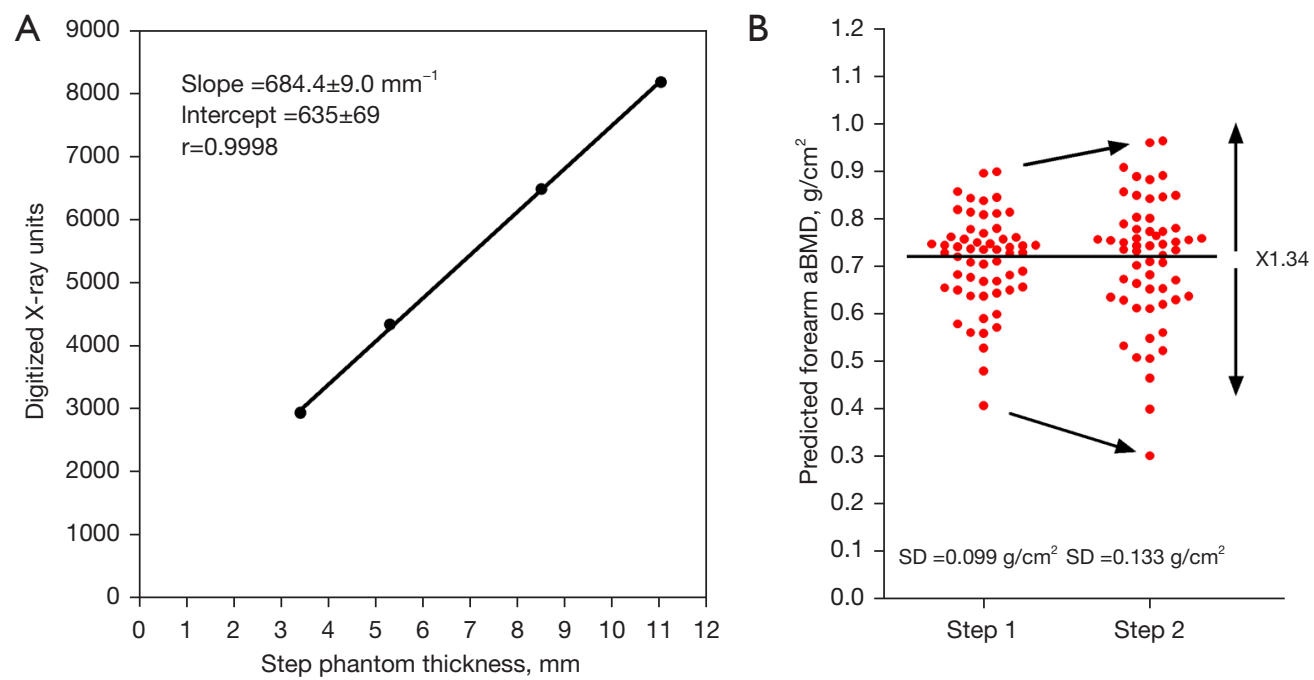

Figure 3 Explanation of the calibration of the Xbone images. (A) Example of a calibration curve obtained with the 4-step phantom. The straight line through the points is the linear regression fit to the data. (B) Explanation of how the digital X-ray predicted forearm aBMD measurements were rescaled to obtain a flat Bland-Altman plot. In Step 1, forearm aBMD was predicted using Eq. [1] with coefficients A, B, and $\mathrm{C}$ determined by a multivariate regression fit to the radius and ulna DXA measurements in the training set. However, for these aBMD estimates the Bland-Altman plot had a slope with a statistically significant correlation coefficient $(\mathrm{r}=-0.57$; $\mathrm{P}<0.0001)$. In Step 2, the slope of the Bland-Altman plot was set to 0 by adjusting each digital X-ray predicted aBMD measurement by first subtracting the mean predicted aBMD of $0.711 \mathrm{~g} / \mathrm{cm}^{2}$ (shown by the horizontal black line) and then multiplying the difference by a scaling factor of 1.34 before adding back the scaled up differences to the mean predicted aBMD. The scaling factor of 1.34 increased the SD of the digital X-ray predicted aBMD measurements from 0.99 to $0.133 \mathrm{~g} / \mathrm{cm}^{2}$ to match the SD of the DXA measurements. DXA, dual X-ray absorptiometry; aBMD, areal bone mineral density; SD, standard deviation.

Instead, we hypothesized a linear relationship of the form, as follows:

$$
\begin{aligned}
\text { DXA aBMD }= & \mathrm{A} * \text { Bone ROI Eq. SPT } \\
& +\mathrm{B} * \text { Soft tissue ROI Eq. SPT }+\mathrm{C}
\end{aligned}
$$

where DXA aBMD (units: $\mathrm{g} / \mathrm{cm}^{2}$ ) was the iDXA aBMD measurement (radius or ulna) at the one-third radius site, bone and soft tissue ROI equivalent step phantom thickness (Eq. SPT) (also radius or ulna) were the Eq. SPT measurements (units $\mathrm{mm}$ ) at the same site obtained from the calibrated digital $\mathrm{X}$-ray images, and $\mathrm{A}, \mathrm{B}$, and $\mathrm{C}$ were constants determined from the measurements of the training cohort. The rationale for this equation is discussed in the Appendix 1.

In the first step of the analysis, digital X-ray radius and ulna measurements in the training cohort in units of Eq. SPT were pooled and analyzed using multivariate least squares regression to determine the best fitting values of the coefficients A, B, and C in Eq. [1] to predict the DXA of forearm aBMD. However, although the mean values of the digital X-ray predicted aBMD and the DXA aBMD were equal at $0.711 \mathrm{~g} / \mathrm{cm}^{2}$, the standard deviation (SD) of the digital X-ray predicted aBMD was smaller (0.099 vs. $0.133 \mathrm{~g} / \mathrm{cm}^{2}$ ), and, as a result the slope of the BlandAltman plot, was statistically significantly different from $0(\mathrm{r}=-0.57 ; \mathrm{P}<0.0001)$. For this reason, a second step was added to the analysis to obtain a flat Bland-Altman plot. The mean aBMD of $0.711 \mathrm{~g} / \mathrm{cm}^{2}$ was subtracted from each digital $\mathrm{X}$-ray-predicted aBMD measurement and the difference was multiplied by a scaling factor of $0.133 / 0.099$ $=1.34$ (Figure $3 B$ ). When the scaled-up differences were added back to the mean aBMD of $0.711 \mathrm{~g} / \mathrm{cm}^{2}$, the slope of the revised Bland-Altman plot was 0. Finally, the scaling factor was used to calculate adjusted values of $\mathrm{A}, \mathrm{B}$, and C that ensured that Eq. [1] returned values of predicted DXA forearm aBMD with a flat Bland-Altman plot. The scaling adjustment shown in Figure $3 B$ did not change the correlation coefficient between the digital $\mathrm{X}$-ray predicted aBMD and DXA aBMD but did slightly increase the $95 \%$ limits of agreement (LOA) of the Bland-Altman plot. 
The digital X-ray-predicted aBMD measurements at the radius and ulna in the validation cohort were calculated using Eq. [1] with values of the adjusted coefficients A, B, and $\mathrm{C}$ determined from the training cohort as described above.

\section{Statistical analysis}

Statistics Kingdom statistical software (Statistics Kingdom, Melbourne, Australia) was used for data analysis (https:// www.statskingdom.com/index.html). The Shapiro-Wilk test was used to evaluate data for normality. Summary statistics were reported as mean \pm SD for normally distributed variables and as median and range for nonnormal variables. Comparisons between the training and validation cohorts were performed using Student's $t$-test for normally distributed variables and the Mann-Whitney test for non-normal variables. Scatter and Bland-Altman plots were drawn to compare the digital X-ray and DXA measurements. A P value $<0.05$ was considered statistically significant.

\section{Results}

Demographic characteristics of participants in the training and validation sets are compared in Table 1. Calibration curves for the training and validation cohorts were highly linear over the range of thicknesses of the step phantom (Figure $3 A$ ). The median correlation coefficient was $\mathrm{r}=0.9997$ (range, 0.9992 to 1.0000 ) with no statistically significant difference between the training and validation sets $(\mathrm{P}=0.78$; Figure $4 A)$. The calibration of the digital $\mathrm{X}$-ray images was stable with no significant difference between the mean intercept of the calibration curves of the two data sets $(\mathrm{P}=0.94$; Figure $4 B)$. However, there was a small but statistically significant difference of $1.8 \%$ between the 2 data sets in the mean slope of the calibration curves $(\mathrm{P}=0.004$; Figure $4 C)$. In addition, the ulna and radius DXA aBMD measurements were statistically significantly higher in the validation cohort compared with the training set $(\mathrm{P}=0.010$ and $\mathrm{P}=0.045$, respectively) (Figure $4 D)$.

When DXA and forearm equivalent phantom thickness measurements for the training cohort were fitted to Eq. [1] after the adjustment to ensure a flat Bland-Altman plot, the values of $\mathrm{A}, \mathrm{B}$, and $\mathrm{C}$ were 0.1772 [standard error (SE): $0.0230] \mathrm{g} / \mathrm{cm}^{2} / \mathrm{mm},-0.1067$ (SE: 0.0227 ) $\mathrm{g} / \mathrm{cm}^{2} / \mathrm{mm}$, and -0.9573 (SE: 0.2646$) \mathrm{g} / \mathrm{cm}^{2}$, respectively. Figure $5 A$ shows the scatter plot of the DXA aBMD measurements for the training cohort plotted against the digital X-ray estimated aBMD values predicted from Eq. [1]. The correlation coefficient was $\mathrm{r}=0.742(\mathrm{P}<0.001)$, and the $95 \%$ LOA for the Bland-Altman plot was $\pm 0.187 \mathrm{~g} / \mathrm{cm}^{2}$ (Figure $5 B$ ). There was no significant difference between the mean bias of the ulna and radius data $(\mathrm{P}=0.12)$.

When the validation cohort was analyzed using the same values of $\mathrm{A}, \mathrm{B}$, and $\mathrm{C}$ from the training cohort listed above, the correlation coefficient was $\mathrm{r}=0.795(\mathrm{P}<0.001)$ (Figure 5C). For the Bland-Altman plot, the mean bias was $-0.002 \mathrm{~g} / \mathrm{cm}^{2}$, the $95 \% \mathrm{LOA}-0.185$ to $+0.181 \mathrm{~g} / \mathrm{cm}^{2}$, for values $\mathrm{A}, \mathrm{B}$, and $\mathrm{C}$, respectively, and the correlation coefficient $\mathrm{r}=0.279$ $(\mathrm{P}=0.031)$ (Figure $5 D)$. There was no significant difference between the mean bias of the ulna and radius data $(\mathrm{P}=0.064)$. In 1 participant, the ulna measurement was a significant outlier.

\section{Discussion}

In this pilot study, we showed that aBMD measurements in the proximal forearm derived from digital radiographs acquired with an external step phantom were comparable to DXA aBMD measurements at the same ROI. The correlation coefficient between digital X-ray estimated aBMD and iDXA measurements in the validation cohort was similar to the correlations (r: range, 0.469 to 0.97 ) of the forearm aBMD measures at middle and proximal sites amongst different peripheral DXA devices $(15,16)$. Thus, RA has potential for application in a variety of medical settings to expand the use of existing $\mathrm{X}$-ray devices. The technique may also provide a widely available and low-cost method to assess patients at high risk of forearm fractures. The main motivation for using this device was to improve accessibility to bone density examinations, and we now have a project across China to enroll more than 20,000 children for a bone age examination using this device. We included the step wedge phantom in the bone age imaging study to also determine aBMD from the same measurement, and validated this method in our study. Further, the $\mathrm{X}$-ray device is very similar to a standard X-ray machine with the same $\mathrm{X}$-ray exposure method, so the method developed in this study could also be applied on other standard X-ray machines with suitable calibration.

The RA was the first method developed to quantify bone mineral on radiographs by calibrating the image intensity using a step wedge as the standard, and literature describing RA appeared as early as the 1930s (17). However, most previous studies have focused on the metacarpals and 

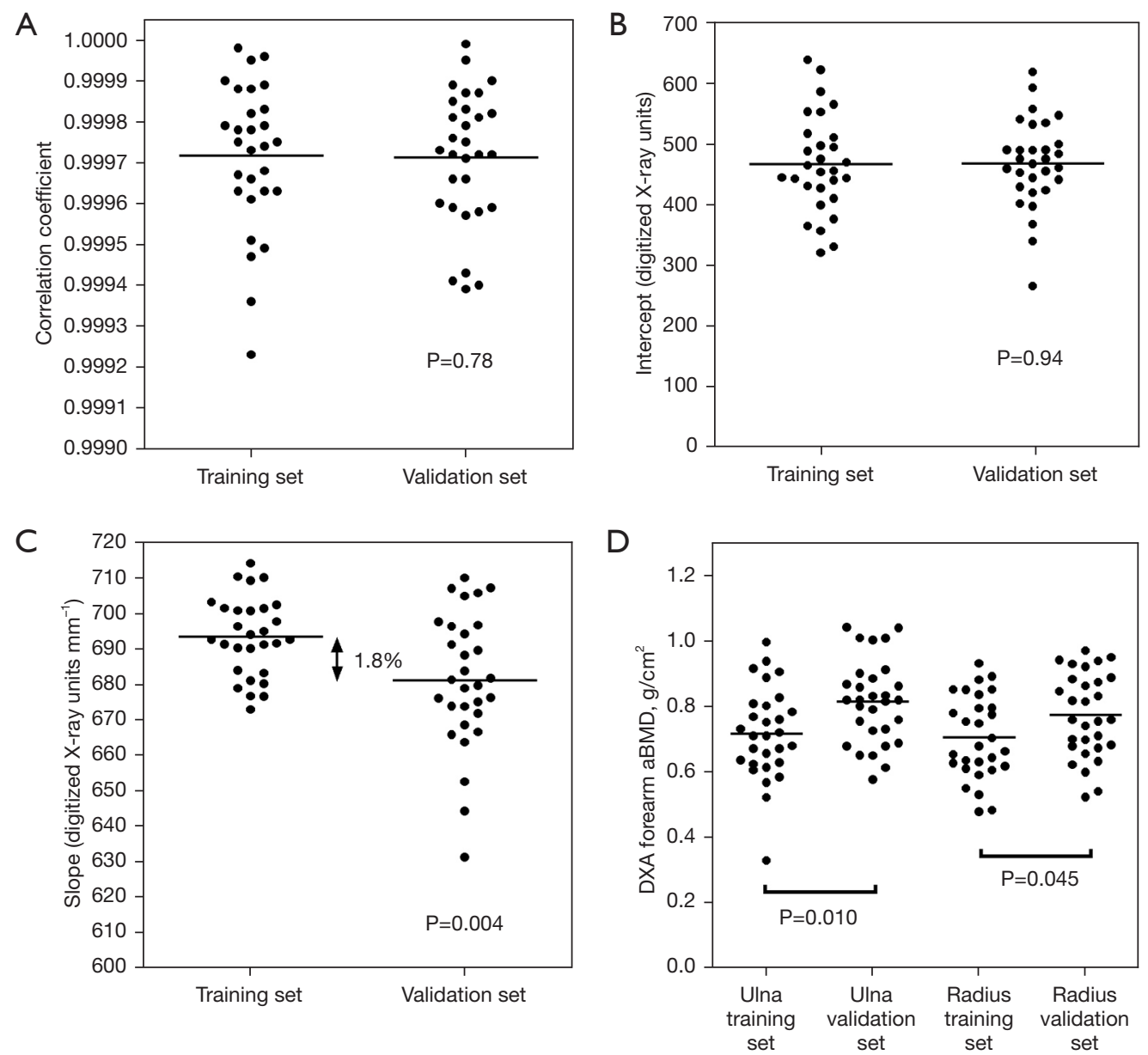

Figure 4 Comparison of step phantom calibration curves and DXA forearm aBMD for the training and validation sets. (A) Plots of the correlation coefficients of the step phantom calibration curves for the training and validation sets; (B) plots of the intercepts of the step phantom calibration curves for the 2 sets; (C) plots of the slopes of the step phantom calibration curves for the 2 sets; (D) plots of the onethird radius forearm ulna and radius iDXA aBMD for participants in the training and validation sets. ROIs were placed 70 mm proximal to the ulna styloid. DXA, dual X-ray absorptiometry; aBMD, areal bone mineral density; ROI, region of interest.

phalanges (18-21). This largely avoided the effect of soft tissue attenuation, which is a confounding factor for RA. Furthermore, aluminum has been the most commonly used wedge phantom material; however, it does not exactly match the X-ray absorption characteristics of bone, and the density of the radiographic image was calibrated in millimeters of aluminum equivalent thickness $(22,23)$. In the present study, we designed a step phantom constructed from a uniform mixture of hydroxyapatite and soft tissue equivalent epoxy resin. The role of the step phantom was to adjust for the variations in the slope and intercept of the calibration curve between exposures so that all digitized forearm measurements were expressed consistently in units of equivalent phantom thickness (Figure $3 A$ ). Variations in the slope and intercept of the calibration curve between exposures and between the training and validation cohorts were small, which affirmed the stability of the Xbone digital X-ray device. A linear equation was used to convert measurements of equivalent phantom thickness into forearm aBMD measurements with coefficients calibrated using ulna and radius aBMD measurements in the training cohort acquired on a GE-Lunar iDXA scanner. The resulting digital $\mathrm{X}$-ray estimates of forearm $\mathrm{aBMD}$ were on the same bone density scale as the iDXA densitometer. Due to differences in calibration, BMD values obtained from different DXA manufacturers are not identical; for example, 

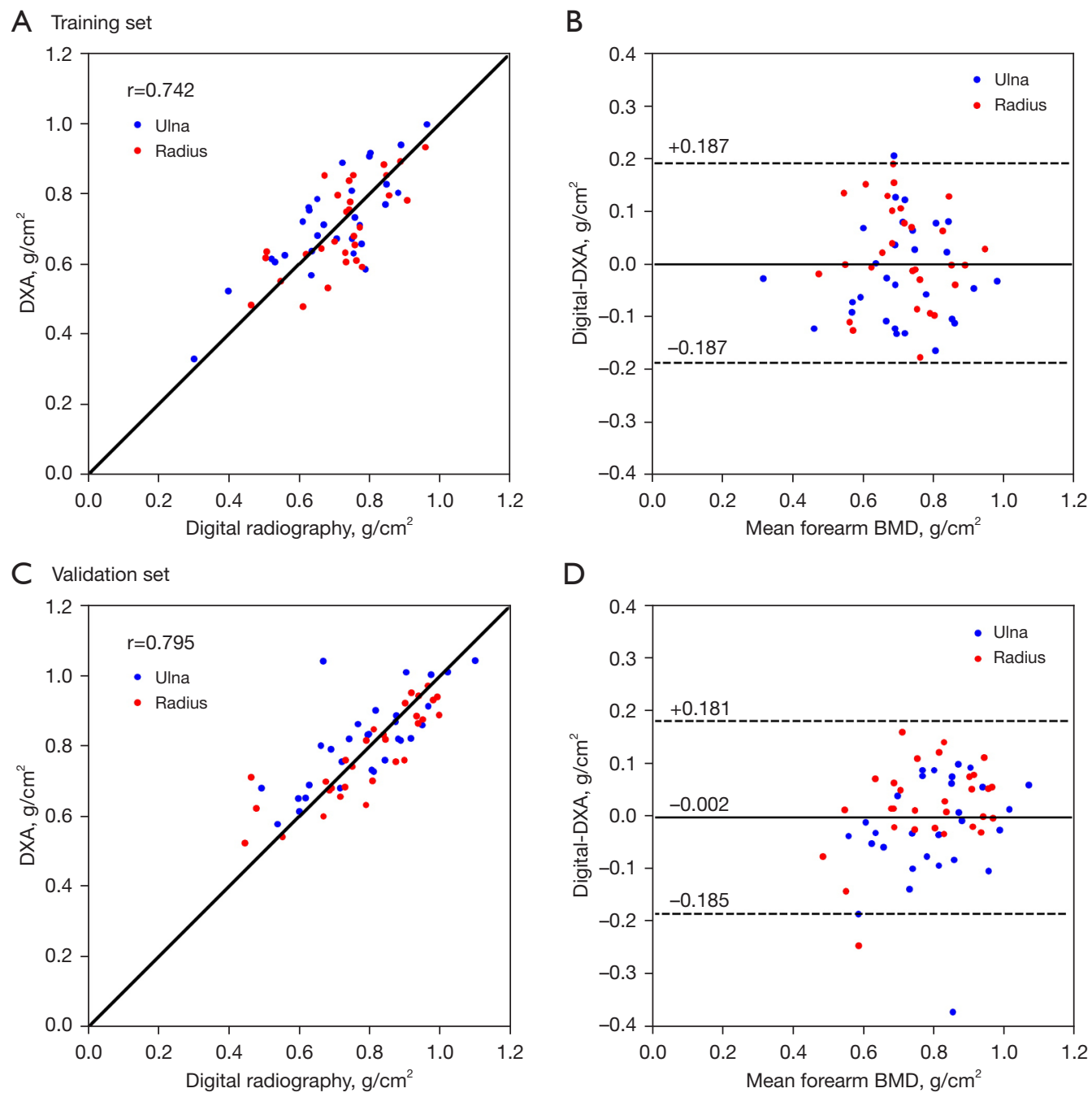

Figure 5 Scatter and Bland-Altman plots for study participants in the training and validation sets. (A) Scatter plot of ulna and radius aBMD for participants in the training set measured by DXA and calculated from digital X-ray measurements using Eq. [1]. The solid line is the line of identity. (B) Bland-Altman plot of the data in (A). Dashed lines show the 95\% LOA. (C) Scatter plot of ulna and radius aBMD for participants in the validation set measured by DXA and calculated from digital X-ray measurements using the same equation. (D) BlandAltman plot of the data in (C). Dashed lines show the 95\% LOA. DXA, dual X-ray absorptiometry; ROI, region of interest; LOA, limits of agreement.

forearm aBMD measurements on Hologic densitometers (Hologic Inc., Marlborough, MA, USA) are around 10\% lower than measurements on GE-Lunar equipment. Measurements of radius and ulnar aBMD have also been obtained from X-rays using the technique of digital X-ray radiogrammetry (DXR). In DXR, cortical porosity and an estimation of the bone volume per projected bone area (VPA) were used. In analogy with Eq. [1], DXR was also empirically calibrated to DXA aBMD.

The RA method has potential to be used in an opportunistic approach to identify forearm bone status obtained from clinical wrist or forearm X-ray examinations. However, caution is necessary since a step phantom is required to calibrate the digital $\mathrm{X}$-ray measurements and the image acquisition parameters need to be standardized with the same settings of appropriate $\mathrm{kV}$, mAs, long focus-film distances, and acquisition time. The Xbone $\mathrm{X}$-ray device used in this study was designed for bone age examination in children with strict radiation exposure protection. The transferability of the present calibration to other similar 
digital X-ray units is not known. In general, images acquired on other digital $\mathrm{X}$-ray devices and at different $\mathrm{kV}$ settings will require calibration with their own training set. Eq. [1] is a simplified approximation to a complex problem in radiation physics. The particular values of the 3 coefficients $\mathrm{A}, \mathrm{B}$, and $\mathrm{C}$ derived here represent a linear approximation of a more general solution that cannot be written as an analytical equation. Their values would be expected to vary with the mean forearm aBMD and forearm thickness of the study population and also with exposure factors such as $\mathrm{kV}$ and filtration. Hence, different coefficients would be required, for example, for a pediatric population.

The correlation coefficients between digital X-ray and DXA aBMD values at the forearm in this study (training set: $r=0.742$; validation set: $r=0.795)$ were similar to the results $(\mathrm{r}=0.77)$ at similar sites in a previous study (24). Most previous RA studies, however, have focused on the hand bones because of the thin layer of overlying soft tissue and minimal beam hardening effect $(18-21,23,25,26)$. The correlation coefficients $(r=0.68,0.775)$ between RA aBMD at phalanges sites and DXA measurements at the forearm in other reports were similar to our results $(19,21)$. In another study, the correlation $\left(\mathrm{r}^{2}=0.81\right)$ between hand BMD measured by RA and middle phalangeal BMD measured by DXA was higher than the outcomes in our study (23). Notably, the DXA measurements in this latter study were not made using standard equipment, but were conducted with a low- and high-energy digital X-ray image pair with image processing analysis.

Since the peripheral skeleton has a different proportion of cortical and trabecular compartments, the changes of bone density with age are different (27). It is possible that bone density of the spine or hip will be normal in a patient with low bone mass at the forearm. An assessment of bone status at the peripheral site may be clinically significant in this circumstance when the specific forearm fracture risk is best evaluated based on a low radius/ulna aBMD measurement. The DXA equivalent forearm aBMD based on our digital X-ray method may improve the approach to osteoporosis testing and identify individuals at risk of fracture. Previous studies have shown that forearm DXA aBMD measurements are associated with spine or hip osteoporotic fractures $(4,11,28,29)$. In the multicenter Study of Osteoporotic Fractures (SOF), Stone et al. showed that the radius aBMD predicted hip and spine fracture with hazard ratios (HR) of 1.54 and 1.73 , respectively (29). Furthermore, the method in our study could help to quantitatively assess the newly formed regenerate bone strength in terms of aBMD during limb lengthening in distraction osteogenesis. Due to fixation metal artifacts and access limitations, state-of-the-art methods like quantitative computed tomography (QCT) and DXA are not appropriate tools to quantitatively determine regenerated bone density.

Our study has several limitations. First, a validation study with 29 participants is relatively small, and further measurements over a longer period of time would better determine the LOA with conventional forearm DXA and the long-term stability of the training set calibration. Second, we did not measure the precision error of the digital X-ray technique. The small precision error (typically $1 \%$ for forearm sites) is one of the major advantages of DXA measurements. Finally, the ulna and radius ROIs placed on the digital X-ray images were drawn by hand, and the future use of automated software might improve the accuracy of these measurements.

\section{Conclusions}

Digital X-ray estimates of proximal forearm aBMD corrected for soft tissue attenuation correlated with DXA measurements with correlation coefficients comparable to those seen for other peripheral bone densitometry technologies. Our method might make measurements of forearm aBMD more widely available in communities with limited access to DXA.

\section{Acknowledgments}

Funding: This work was supported in part by Beijing Hospitals Authority Youth Programme (QMS20200402), Beijing Jishuitan Hospital Elite Young Scholar Programme (XKGG201805), Beijing Municipal Health Commission (BMHC2019-9), the National Natural Science Foundation of China (81901718, 81771831), Beijing Hospitals Authority Clinical Medicine Development of Special Funding Support (ZYLX202107), and the Wellcome/EPSRC Centre for Medical Engineering (WT 203148/Z/16/Z).

\section{Footnote}

Reporting Checklist: The authors have completed the TRIPOD reporting checklist. Available at https://qims. amegroups.com/article/view/10.21037/qims-21-842/rc

Conflicts of Interest: All authors have completed the ICMJE 
uniform disclosure form (available at https://qims. amegroups.com/article/view/10.21037/qims-21-842/coif). Klaus Engelke is a part-time employee of BioClinica, Inc. The other authors have no conflicts of interest to declare.

Etbical Statement: The authors are accountable for all aspects of the work and ensuring that questions related to the accuracy or integrity of any part of the work are appropriately investigated and resolved. The study was conducted in accordance with the Declaration of Helsinki (as revised in 2013). The study was approved by the institutional review board of the principal investigator's hospital, and all participants provided their written informed consent.

Open Access Statement: This is an Open Access article distributed in accordance with the Creative Commons Attribution-NonCommercial-NoDerivs 4.0 International License (CC BY-NC-ND 4.0), which permits the noncommercial replication and distribution of the article with the strict proviso that no changes or edits are made and the original work is properly cited (including links to both the formal publication through the relevant DOI and the license). See: https://creativecommons.org/licenses/by-nc-nd/4.0/.

\section{References}

1. Cheng X, Zhao K, Zha X, Du X, Li Y, Chen S, et al. Opportunistic Screening Using Low-Dose CT and the Prevalence of Osteoporosis in China: A Nationwide, Multicenter Study. J Bone Miner Res 2021;36:427-35.

2. Cheng X, Yuan H, Cheng J, Weng X, Xu H, Gao J, Huang M, Wáng YXJ, Wu Y, Xu W, Liu L, Liu H, Huang C, Jin Z, Tian W; Bone and Joint Group of Chinese Society of Radiology, Chinese Medical Association (CMA), Musculoskeletal Radiology Society of Chinese Medical Doctors Association, Osteoporosis Group of Chinese Orthopedic Association, Bone Density Group of Chinese Society of Imaging Technology, CMA*. Chinese expert consensus on the diagnosis of osteoporosis by imaging and bone mineral density. Quant Imaging Med Surg 2020;10:2066-77.

3. Samelson EJ, Broe KE, Xu H, Yang L, Boyd S, Biver $\mathrm{E}$, et al. Cortical and trabecular bone microarchitecture as an independent predictor of incident fracture risk in older women and men in the Bone Microarchitecture International Consortium (BoMIC): a prospective study. Lancet Diabetes Endocrinol 2019;7:34-43.
4. Cummings SR, Black DM, Nevitt MC, Browner W, Cauley J, Ensrud K, Genant HK, Palermo L, Scott J, Vogt TM. Bone density at various sites for prediction of hip fractures. The Study of Osteoporotic Fractures Research Group. Lancet 1993;341:72-5.

5. Matkovic V, Landoll JD, Badenhop-Stevens NE, Ha EY, Crncevic-Orlic Z, Li B, Goel P. Nutrition influences skeletal development from childhood to adulthood: a study of hip, spine, and forearm in adolescent females. J Nutr 2004;134:701S-5S.

6. Ducher G, Prouteau S, Courteix D, Benhamou CL. Cortical and trabecular bone at the forearm show different adaptation patterns in response to tennis playing. J Clin Densitom 2004;7:399-405.

7. Macdonald HM, Nishiyama KK, Hanley DA, Boyd SK. Changes in trabecular and cortical bone microarchitecture at peripheral sites associated with 18 months of teriparatide therapy in postmenopausal women with osteoporosis. Osteoporos Int 2011;22:357-62.

8. Cheung AM, Majumdar S, Brixen K, Chapurlat R, Fuerst T, Engelke K, Dardzinski B, Cabal A, Verbruggen N, Ather S, Rosenberg E, de Papp AE. Effects of odanacatib on the radius and tibia of postmenopausal women: improvements in bone geometry, microarchitecture, and estimated bone strength. J Bone Miner Res 2014;29:1786-94.

9. Miller PD, Siris ES, Barrett-Connor E, Faulkner KG, Wehren LE, Abbott TA, Chen YT, Berger ML, Santora AC, Sherwood LM. Prediction of fracture risk in postmenopausal white women with peripheral bone densitometry: evidence from the National Osteoporosis Risk Assessment. J Bone Miner Res 2002;17:2222-30.

10. Giraudo C, Cavaliere A, Lupi A, Guglielmi G, Quaia E. Established paths and new avenues: a review of the main radiological techniques for investigating sarcopenia. Quant Imaging Med Surg 2020;10:1602-13.

11. Blake GM, Fogelman I. Fracture prediction by bone density measurements at sites other than the fracture site: the contribution of BMD correlation. Calcif Tissue Int 2005;76:249-55.

12. Zhao Y, Wang W, Cheng X. Optimal Selection of Exposure Parameters of Orthogonal Transmission Target Tube in Children's Bone Age DR Examination of Wrist (in Chinese). China Medical Equipment 2021;36:67-70.

13. Zhao J, Xing Y, Zhou Q, Jin W, Wacker W, Barden HS. Can forearm bone mineral density be measured with dxa in the supine position? A study in Chinese population. J Clin Densitom 2010;13:147-50. 
14. Radiation Protection Service UoL. Radiation Risk Assessment 22: GE Lunar iDXA Bone Densitometer (updated 21/09/2018). Accessed 2021 November 16. Available online: https://wsh.leeds.ac.uk/download/ downloads/id/519/x-rays_-_ge_idxa.pdf

15. Shepherd JA, Cheng XG, Lu Y, Njeh C, Toschke J, Engelke K, Grigorian M, Genant HK. Universal standardization of forearm bone densitometry. J Bone Miner Res 2002;17:734-45.

16. Hazell TJ, Vanstone CA, Rodd CJ, Rauch F, Weiler HA. Bone mineral density measured by a portable X-ray device agrees with dual-energy $\mathrm{X}$-ray absorptiometry at forearm in preschool aged children. J Clin Densitom 2013;16:302-7.

17. 5. X-ray Absorptiometry. J ICRU 2009;9:37-58.

18. Gulam M, Thornton MM, Hodsman AB, Holdsworth DW. Bone mineral measurement of phalanges: comparison of radiographic absorptiometry and area dual X-ray absorptiometry. Radiology 2000;216:586-91.

19. Yang SO, Hagiwara S, Engelke K, Dhillon MS, Guglielmi G, Bendavid EJ, Soejima O, Nelson DL, Genant HK. Radiographic absorptiometry for bone mineral measurement of the phalanges: precision and accuracy study. Radiology 1994;192:857-9.

20. Hagiwara S, Engelke K, Takada M, Yang SO, Grampp S, Dhillon MS, Genant HK. Accuracy and diagnostic sensitivity of radiographic absorptiometry of the second metacarpal. Calcif Tissue Int 1998;62:95-8.

21. Takada M, Engelke K, Hagiwara S, Grampp S, Jergas M, Glüer CC, Genant HK. Assessment of osteoporosis: comparison of radiographic absorptiometry of the phalanges and dual X-ray absorptiometry of the radius and lumbar spine. Radiology 1997;202:759-63.

Cite this article as: Wang L, Huang $\mathrm{P}, \mathrm{Du} \mathrm{H}$, Geng J, Yin X, Liu Y, Puri T, He B, Lyu L, Cheng X, Jiang X, Engelke K, Blake GM. Assessment of bone densitometry using radiography with a step-wedge phantom: a pilot study of the forearm. Quant Imaging Med Surg 2022;12(6):3340-3350. doi: 10.21037/qims-21842
22. Kolbeck S, Bail H, Weiler A, Windhagen H, Haas N, Raschke M. Digital radiography. A predictor of regenerate bone stiffness in distraction osteogenesis. Clin Orthop Relat Res 1999;(366):221-8.

23. Dendere R, Whiley SP, Douglas TS. Computed digital absorptiometry for measurement of phalangeal bone mineral mass on a slot-scanning digital radiography system. Osteoporos Int 2014;25:2625-30.

24. Adami S, Zamberlan N, Gatti D, Zanfisi C, Braga V, Broggini M, Rossini M. Computed radiographic absorptiometry and morphometry in the assessment of postmenopausal bone loss. Osteoporos Int 1996;6:8-13.

25. Matsumoto C, Kushida K, Yamazaki K, Imose K, Inoue T. Metacarpal bone mass in normal and osteoporotic Japanese women using computed X-ray densitometry. Calcif Tissue Int 1994;55:324-9.

26. Cosman F, Herrington B, Himmelstein S, Lindsay R. Radiographic absorptiometry: a simple method for determination of bone mass. Osteoporos Int 1991;2:34-8.

27. Link TM. Osteoporosis imaging: state of the art and advanced imaging. Radiology 2012;263:3-17.

28. Cummings SR, Black DM, Nevitt MC, Browner WS, Cauley JA, Genant HK, Mascioli SR, Scott JC, Seeley DG, Steiger P. Appendicular bone density and age predict hip fracture in women. The Study of Osteoporotic Fractures Research Group. JAMA 1990;263:665-8.

29. Stone KL, Seeley DG, Lui LY, Cauley JA, Ensrud K, Browner WS, Nevitt MC, Cummings SR; Osteoporotic Fractures Research Group. BMD at multiple sites and risk of fracture of multiple types: long-term results from the Study of Osteoporotic Fractures. J Bone Miner Res $2003 ; 18: 1947-54$. 


\section{Supplementary}

We discuss the rationale for adopting Eq. [1] to estimate forearm DXA aBMD (units: $\mathrm{g} / \mathrm{cm}^{2}$ ) from digitized X-ray measurements of attenuation in regions of interest (ROIs) in the radius and ulna and an adjacent soft tissue ROI between the radius and ulna (Figure $2 A$ ). The digitized X-ray measurements were converted into measurements of equivalent step phantom thickness (Eq. SPT; units: $\mathrm{mm}$ ) using the calibration provided by the step phantom (Figure $3 A$ ).

$$
\text { DXA aBMD }=\mathrm{A}^{\star} \text { Bone ROI Eq. SPT }+\mathrm{B}^{\star} \text { Soft tissue ROI Eq. SPT }+\mathrm{C}
$$

The digitized pixel measurements provided by the Xbone device scale with the logarithm of X-ray intensity. Hence the digitized X-ray measurements of the step phantom have a linear relationship with the thickness of each step (or equivalently with their areal density in units of $\mathrm{g} / \mathrm{cm}^{2}$ ) provided confounding technical issues such as the polychromatic X-ray spectrum, beam hardening and scatter are ignored (Figure 3A). To justify Eq. [1], as a first step we represent the attenuation by soft tissue in the bone ROI in the digital X-ray image by its equivalent aBMD (i.e., the areal density of bone mineral that attenuates the $\mathrm{X}$-ray beam to the same degree as the soft tissue). With this understanding we write the digital $\mathrm{X}$-ray equivalent forearm aBMD in the bone ROI due to the combined attenuation by bone and soft tissue as:

$$
\text { Bone ROI digital X-ray equivalent aBMD = P*Bone ROI Eq. SPT }
$$

In Eq. [2], $\mathrm{P}$ is the constant that relates equivalent step phantom thickness measured in $\mathrm{mm}$ to its equivalent areal bone mineral density measured in $\mathrm{g} / \mathrm{cm}^{2}$. To convert from equivalent aBMD due to the combined attenuation by bone and soft tissue and estimate the true bone aBMD that would be measured by DXA we need to subtract the soft tissue contribution from the total digital X-ray attenuation in the bone ROI. To do this we subtracted a fraction $\mathrm{Q}$ of the equivalent step phantom thickness measured in the soft tissue ROI between the radius and ulna (Figure $2 A$ ):

Bone ROI DXA aBMD = P*(Bone ROI Eq. SPT $-Q^{*}$ Soft tissue ROI Eq. SPT $)$

Given that there is less soft tissue in the bone ROI than the adjacent soft tissue ROI due to the replacement of some soft tissue by bone, we expect $\mathrm{Q}<1$. Multivariate regression fits of the training set data to Eq. [3] gave $\mathrm{Q}=0.6$. In reality, Eq. [3] is a simplification of a complex problem and we expect values of $Q$ to vary between individuals depending on bone size and soft tissue thickness through the forearm. As a generalization of Eq. [3] we also examined linear equations that allowed a constant term, R, which gave a better fit to the data:

Bone ROI DXA aBMD = P*(Bone ROI Eq. SPT $-\mathrm{Q}^{*}$ Soft tissue ROI Eq SPT $)+\mathrm{R}$

We therefore arrive at Eq. [1] as providing a suitable fit to the data where A, B and C in Eq. [1] are respectively $\mathrm{P},-\mathrm{P}^{*} \mathrm{Q}$ and $\mathrm{R}$ in Eq. [4].

As emphasized in the Discussion, Eq. [1] is a simplified approximation to a complex problem. Values of the three coefficients A, B and C in Eq. [1] derived here represent a linear approximation to a more complex solution with values that are valid for the mean forearm aBMD and forearm thickness of the study population. 